\title{
Resolution Revolutions on the Cryo-EM Structures of Ribosome- A Study: 1981-2018
}

\author{
Arita Acharjee ${ }^{1 *}$ and Ananya Chatterjee ${ }^{2}$ \\ ${ }^{1}$ International Institute of Innovation and Technology, DH-6/24, Street No. 0317, Action Area- ID, New Town, Kolkata- 700156. \\ ${ }^{2}$ Department of Biological Sciences, Indian Institute of Science Education and Research- Kolkata, Mohanpur, Nadia, West Bengal, India.
}

\begin{abstract}
Ribosomes are large ribonucleoprotein complexes that have been proved to be ideal biological sample for the development of the cryo-Electron Microscopy (cryo-EM) field. On the other hand, advancement in cryo-EM technologies have helped in elucidating detailed structure-function aspects of various kinds of ribosomes, belonging to prokaryotic, organellar, and complex eukaryotic systems. In a relatively short duration of time, through rapid technological advances, cryo-EM has generated number of ribosome structures from sub atomic to atomic resolutions with the capability of visualizing the side chain interactions of protein structures. This has given us the scope to visualize various drugs and their binding sites on the ribosomes, wherefrom there are greater chances to improve further and modify the drugs and verify their efficacy. This review provides an account of the improvement of the cryo-EM in the light of performing research on ribosomes and understanding some of the ribosome's structurefunction aspects in further details.
\end{abstract}

Key Words: Cryo-electron microscopy, ribosome, single particle reconstruction, Direct Electron Detectors, Image processing.

\section{Introduction}

Ribosomes form one of the main bases of life as they are the molecular manufacturing centres for the production of cellular proteins that in turn regulate major chemical processes of the cell, maintain integrity, helps in damage repair and other barrage of cellular activities that help in making the cell viable and running. Thus, translation, or, protein synthesis, in prokaryotes and eukaryotes alike, is one of the crucial and fundamental molecular mechanisms of the cell, in which peptide chains are synthesized from the triplet codons present in messenger RNA (mRNA) with the help of transfer RNA (tRNA) and the entire process is regulated and executed within the master manufacturer- the ribosome.
Ribosomes are complex macromolecular machines consisting of ribosomal RNAs (rRNAs) and proteins. The ribosome recruits corresponding stage factors (initiation, elongation, release and recycling) for precise and immaculate execution of translation and each of these factors in consortium with the ribosome, forms definitive complexes dedicated to carry out their designated actions efficiently and meticulously, rendering the movement and speed of this process to be highly complex yet accurate. Ribosome is the essential platform of this process as the codons are read and the synthesis of peptide chain occurs here. Essentially both the free and bound

\footnotetext{
* Address of Correspondence: Ms. Arita Acharjee.

Affiliation: DH-6/24, Street No. 0317, Action Area- ID, International Institute of Innovation and Technology, New Town, Kolkata- 700156.WB, India.

Email address: arita.acharjee27@gmail.com; aritaacharjee@i3tk.org
} 
ribosomes (organellar) are protein synthesis machineries consisting of two subunits -the small subunit and the large subunit, which differ in mass and size in both prokaryotic and eukaryotic organisms. The prokaryotic ribosomes are made up of $30 \mathrm{~S}$ and 50S subunits, which, when coming together make the 70S ribosome whereas in eukaryotic organisms, the total $80 \mathrm{~S}$ ribosome is made up of $40 \mathrm{~S}$ and $60 \mathrm{~S}$ subunits. The sedimentation coefficients considerably vary among certain classes of organellar ribosomes.

Ribosomes have three sites for tRNA, the A site (aminoacyl), which accepts the incoming aminoacyl- tRNA; the P site (peptidyl), which holds the tRNA with the nascent peptide chain; and E site (exit), which holds the deacylated tRNA before it leaves the ribosome. In prokaryotes, the $30 \mathrm{~S}$ subunit binds mRNA and the anticodon stem-loops of remarkably conserved nature of critical regions of the tRNA, and contributes to the fidelity of translation by monitoring base pairing between codon and anticodon in the decoding process. The 50S subunit binds the acceptor arms of tRNA and catalyzes peptide bond formation between the incoming amino acid on A-site tRNA and the nascent peptide chain attached to the P-site tRNA. Both subunits are involved in translocation, in which the tRNAs and mRNA move precisely through the ribosome, one codon at a time (47). Understanding the structural attributes of ribosome, thus can give a clearer idea about the protein synthesis process which is of utter importance in medicinal studies as many drugs and antibiotics target the protein synthesis pathway as well as their machinery (10).

Though the overall scheme of translation was elucidated many years ago, there were difficulties in a detailed structural analysis of ribosomal features as ribosomes were considered to be too large for high resolution structural analyses. Another drawback which was present is that the biochemical and genetic tools were not that much developed and definitely not enough to understand this complex structure. As a result, translation took a back seat to other problems in molecular biology after its golden age in the 1960s and 70s. Progress is attained with the application of crystallography techniques, when single particle reconstruction technique emerged. In light of the highly advanced and precise technique of cryo-EM, the amount of sample needed was also much less than crystallography (24). Therefore, cryo-EM revolutionized structural analyses of ribosomes in complex with tRNA, mRNA and also with other factors, which are crucial determining factors in regulatory mechanisms of ribosomes $(5,27)$.

Electron microscopy of biological specimens has developed manifolds since its days of inception to image bacteriophage, as done by Ruska (1941) (49). As electron beam is used to visualize the samples, the major difficulty in cryo-EM of biological samples is the radiation damage from the breakage of chemical bonds. Another problem faced was the requirement of high vacuum in the beam path as electron gets scattered in air as vacuum comprises the structural integrity of biological samples in aqueous environment. Cryo-EM came to the rescue of these problems by introducing the concept of amorphous ice which is the noncrystalline form of solid water. This method as conceptualized by Dubochet et al. (1981) (14) was a major step to overcome a setback. Artifacts formed from dehydration or fixation techniques were also reduced and the flash freezing method preserved the samples in a closer to native state. This therefore helped in understanding the native structures of the biological samples. Another aspect in case for cryo-EM that makes it stand out of the crowd is the ability to capture the essence of dynamicity of the complex. Dynamic particles are quite stubborn and create hurdles in crystal formation but cryo-EM is the answer in face of those obstacles. There is an ever-changing pattern of ribosomal conformation due to accommodation of / association with other factors or particles in specialised cases of pathogenic protein synthesis in bacteria, parasites or other pathogens or for essential binding affinity to specific drug-delivery. All these reasons made it all the very important target that needs to be studied from all structural aspects. Hence, cryoEM is one of the best methods to elucidate the intricacies (42) of dynamicity, binding affinity, residual interactions and other individual or associative/dissociative characteristics of ribosomes. 
From the year 2000 to until 2013 the resolutions of the cryo-EM structures of many asymmetric biological complexes including ribosome complexes were generally in the range of $14 \AA$ to $6 \AA$. However, 2013 onwards, during the last four to five years, very rapidly several improvement happened in the cryo-EM field that led to what is called "resolution revolution", wherein, people started solving cryo-EM structures of various complexes and proteins in the scale of atomic resolutions i.e., $\sim 3.5 \AA$ or better, and ribosomes have been no exception to this race (see Figure 1).

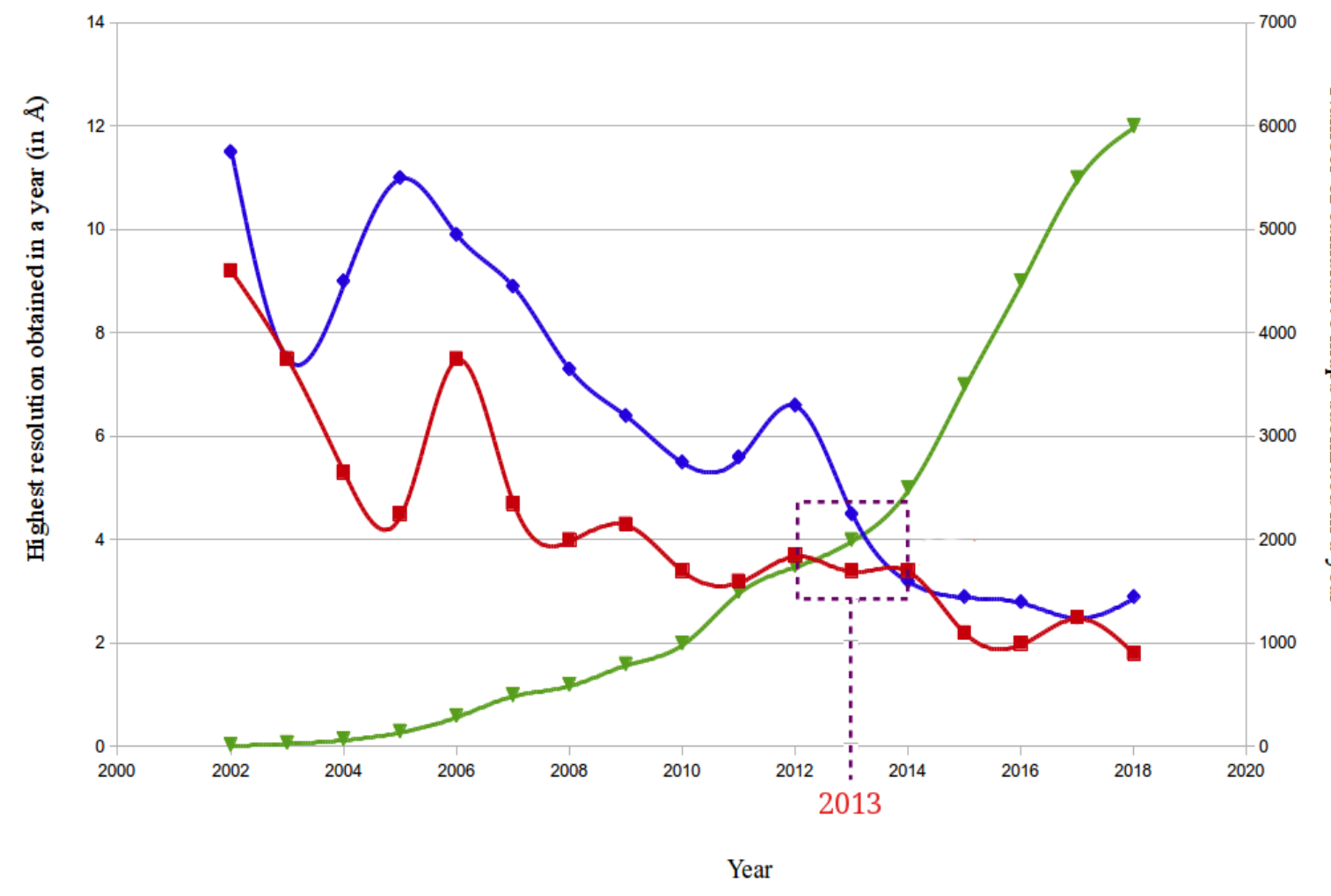

Figure 1: Resolution revolution in cryo-EM from 2002-2018.

The red line depicts the improvement in resolution (with lesser angstrom levels reached) with progressive years (2002-2018) and the green line depicts the cumulative maps deposite to EMDB throughout the span of 2002-2018. The blue line highlights solely on the improvement of resolution (with lesser angstrom levels reached) of ribosomes with progressive years (2002-2018). [Sourced from EMDB Database].

The triad junction as boxed in the figure reflects the timeline in 2013, when the cumulative number of maps submitted to EMDB per year shoots up along with drastic improvement in resolution of both ribosomal and overall particles reconstructed using cryo-EM. 


\section{What formed the basis of the advancement in cryo-EM?}

\section{Technical enhancements of image capture, inception of Direct Electron Detectors and subsequent improved image processing}

Because of the occurrence of low electron dense atoms, biological samples are subjected to radiation damage under the high velocity electron beam in the TEM, resulting in significant loss of signals which ultimately culminates to noisy data. This demands that single particle cryo-EM performs class averaging of a fairly large number of low dose images of the same sample to gain better signal and improve signal to noise ratio. Compilation of averaged out data to reconstitute 3D images of the sample particles warrant that aberrations, framework, boundaries or limitations of the microscope be determined for each micrograph along with orientation and translational aspects for each individual good particle image (43). The process and mechanism of sample preparation, image capture, image processing and structural analyses for 3D reconstruction of particles has been schematically elucidated in Figure 2. Two crucial limiting factors that hindered the progress and improvement of the microscopic quality have been the image-recording medium condition and image blurring. These were caused owing to instability of the sample stage or incident electron beam induced motion. High signal-to-noise ratio (SNR) enhances the image quality quite significantly but the limiting factor is- how accurate has been the calculation for the individual yet crucial factors, where the image detector has an important role to play. To significantly enhance and improve image clarity and reduce unnecessary background signal-tonoise ratio, recording high-resolution information by direct detection process became immensely important.

So far, the electron image recording media had been dominated by either photographic films or scintillator-based chargecoupled device (CCD) cameras. However, they do not satiate the requirement for achieving very high-resolution image. The recent development of a new generation of complementary metal-oxide-semiconductor (CMOS) based direct electron detectors that bypass most of the adversities can collect images with superior resolutions. These new sensors are capable of direct detection of incoming electrons in the silicon without a scintillator, with enhanced detective quantum efficiency (DQE) at high frequency. Recent reports mention to have characteristic high performance DE-12 camera from Direct Electron (LP, San Diego, CA). The 3D reconstruction calculated from the data recorded by this camera has been found to reach nearer to its Nyquist limit.

Image blurring as a consequence of electron beam induced motion has posed to be one of the major limiting factors to high-end resolution when frozen hydrated biological samples were imaged. In practice, beam induced motion and the resultant deteriorated image quality in terms of resolution and clarity seemed impossible to circumvent using the then existing cryo-EM techniques. The high frame rate of new CMOS based (10 40 frames/sec) Direct Electron Detectors (DED) hereby provides a way of correction for such image blurring by recording a "movie" throughout the span of exposure. That is, a single exposure is broken down/divided into a number of subframes within adequate short duration in order to freeze the motion to the set/standard level. The setback here, in spite of increasing propensity of signal, is the constant camera readout noise on each subframe. Even so, in case of large macromolecular complexes like viruses or ribosomes particles, the subframes were preferably used as independent images for refinement leading to increased clarity and better resolution. However, for smaller objects, the ability to accurately determine particle orientation becomes difficult due to low SNR of each particle within a single subframe. To find for a reasonable solution, further modification of detector reducing both detection and readout noise was considered as a compromise, keeping beam induced motion corrections limited to either the whole frame or large portions of a frame (see Figure 3). 


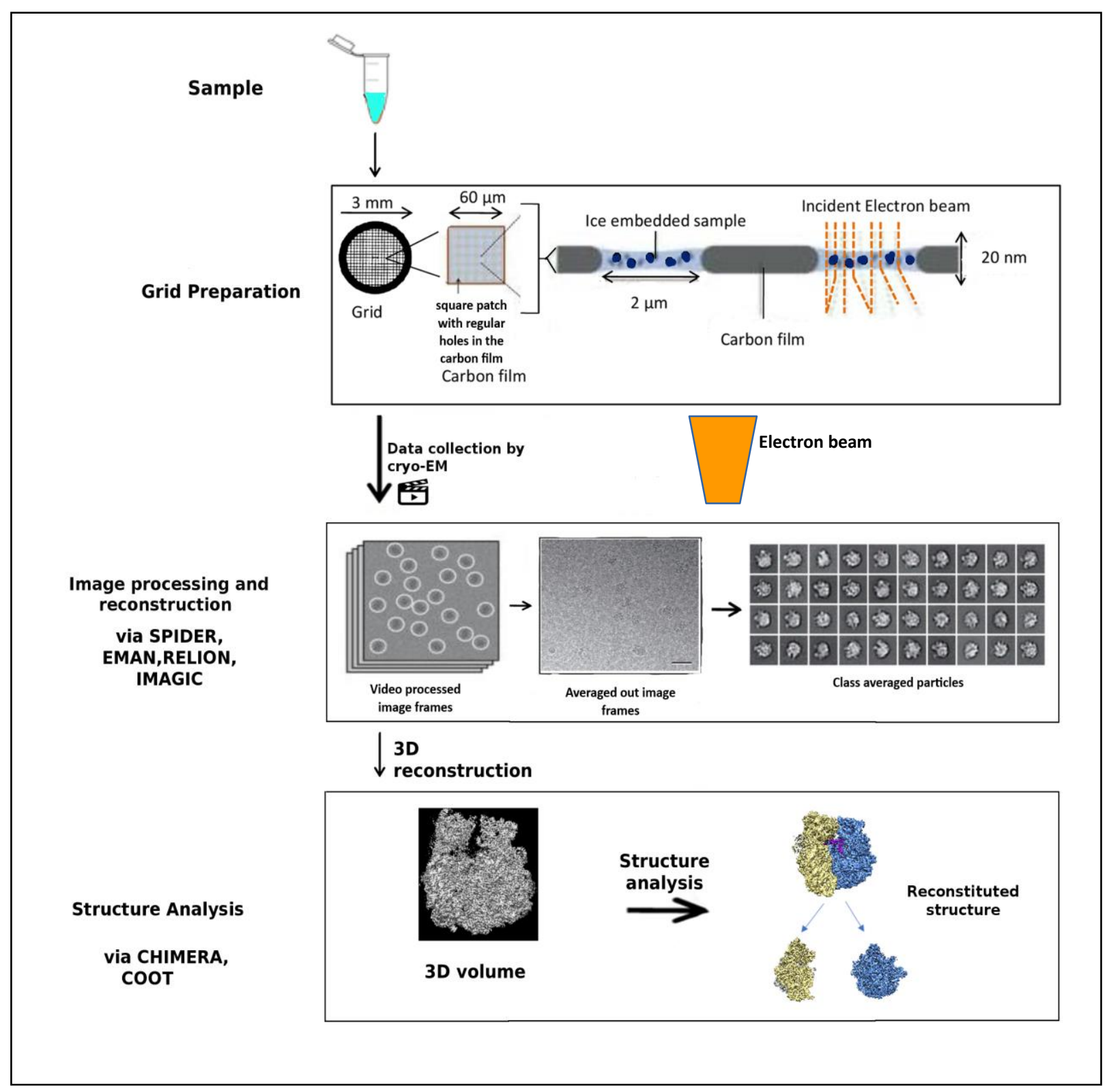

Figure 2: Schematic representation of the process of sample preparation, image capture, data collection, image processing and structural analyses of single particle ribosomes by cryo-EM.

\section{Improvements in image processing}

Recent modifications and developments in image processing along with the new detectors, have equally contributed in this field to obtain better reconstructions and thereby better resolutions. The first development aims at solving the difficulties in handling structurally heterogeneous samples whereas the second addresses the problem of the irradiating electron beam induced movements of the sample. Both synergistically have prevented loss in information and resulted in better resolution of the reconstructed particles (35). 


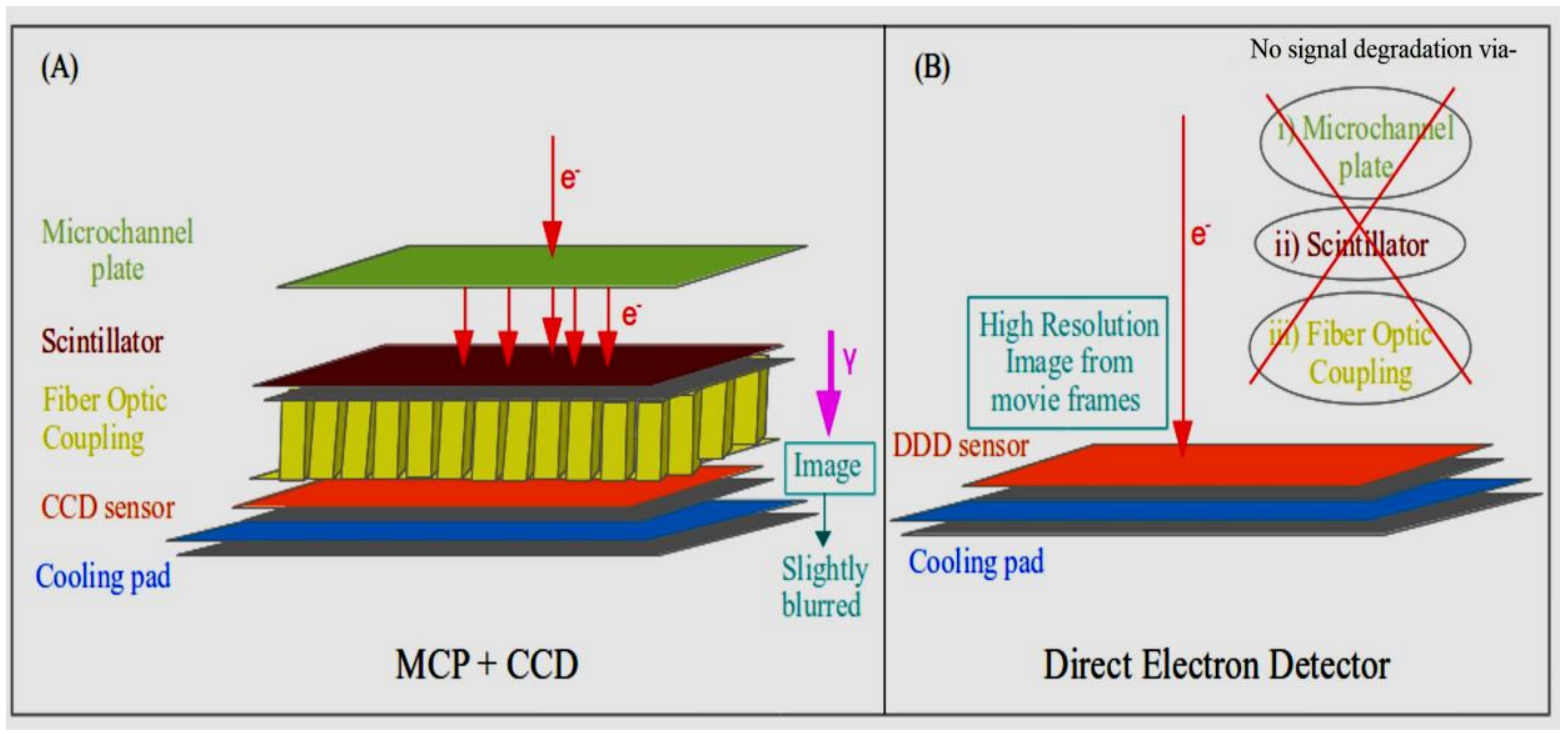

Figure 3: Improvement in resolution of images due to Direct Electron Detector.

(A) Portrays the schematic representation of the image captured by $\mathrm{MCP}+\mathrm{CCD}$, whereas

(B) Depicts high resolution image collection from Direct Electron Detector.

In case of macromolecular complexes possessing unique 3D structures with complex heterogeneity, they will have projections of different structures which are not categorically aligned. From these, the existing structural homogenous subsets were obtained and combined to reconstitute a 3D structure will at the best result in a blurry map. Early 3D classification was done manually, and moreover, it used to give more weightage to structural variations, usually not available, creating more troubles dealing with heterogenous samples. However, a breakthrough for general application 3D reconstruction (non-manual) came about with the introduction of statistical maximum likelihood algorithms based on multivariate statistical analyses. Other contributing factors are variance calculation by bootstrapping method and non-statistical particle refinement. Occasional manual intervention along with Bayesian approach helped in both statistical framework and reduction of noise in IMAGIC, XMIPP, SPIDER $(64,13,60)$ and other softwares came in the flow that changed the face of 3D classification and high-resolution reconstructions via cryo-EM (45).

The second modification again comes at the clause where image processing shares a close-knit bond with the developed new direct detectors (5). Exploiting this connection, beam induced sample movements were considerably reduced via the new movie mode of detectors when Niko Grigorieff and his colleagues realized that realignment of individual movie frames can correct for these motions, reducing the background noise considerably. This in turn rendered into high resolution structures with accurate density fits. The ribosomes (MRC) stand witness to this breakthrough consortium. The ribosomes were reconstituted at a resolution of $4 \AA$ using 35,000 asymmetric particles, displaying even side chain densities for larger amino acids.

With application of the improved techniques and softwares like EMAN, BSoft, RELION, $(39,28,50)$ which brought in better algorithmic solutions, cryo-EM can determine structures now with finer resolutions such as $2.2 \AA$ and $2.3 \AA$, as demonstrated by structure of $465 \mathrm{kDa}$ b-galactosidase (6). Large and relatively stable complexes, ideally ribosomes, proved especially attuned to analysis using cryoEM methods, initially at medium resolution $(41,48)$ and more recently at near- atomic resolution $(2,16,30,67)$. However, most of the near-atomic-resolution structures reported essentially has been of proteins which are categorized as assemblies or complexes or in other words, macromolecular structures. Though cryo-EM technology is primarily recognized for its able and adept detailed structural visualization and analysis of comparatively stable proteins ranging above $150 \mathrm{kDa}$ (61), smaller protein sample visualization by cryo-EM is not anymore beyond the possible horizon. 


\section{The revolution of Single particle cryo-Electron Microscope}

Cryo-EM has provided intricate information about structures which were not comprehensible before. Ribosomes have always proved to be one of the best samples to be studied by cryo-EM, among all other asymmetric molecules. With ribosome as our main highlight, some of the previous studies could be cited which analysed the particle in different domains and in minute details to scoop out the finer mechanistic properties of the particles revealed only at higher resolutions. The accompanying extra information is uncovered now.

Significant contributions by Malhotra et al., 1998 (40) through cryo-EM helps us to visualize the localization of fMet-tRNAfMet along with fitting of L1 protein on Escherichia coli 70S ribosome, unambiguously complementing the corresponding X-ray structure indicates the then increase in superresolving power of cryo-EM. Other than this group, Valle et al. in 2002 (62) explored via cryo-EM, as to how the tRNA anti-codon arm conform differently for apparent facilitation of codon-anticodon interaction. Moreover, they even scrutinized how association of tRNA with 50S GTPase centre on ribosome can be suggestive of its active role in prompting signal for GTP hydrolysis, post codon recognition. In order to understand E. coli $70 \mathrm{~S}$ ribosome to a greater extent, other notable works such as that of Datta et al. in 2005 (12) delved a little deep into identifying and analysing ribosomal translocation process. Using cryo-EM as the tool, they demonstrated an arc-like connection (ALC) which joins EF-G, G' domains and L7/L12 stalk of 50S.GDP subunit, delineating the boundary with the help of undecagold tag at the tip of EFG-G' domain. The GDP and GTP conformational changes thus elucidated the movement, process of translocation and the key players involved in the ALC formation. After elongation process, the initiation pathway and its players did not take a back seat either. In 2016, Hussain et al (29) demonstrated how the initiation factors and their roles could be demonstrated by the process of reconstituting 11 single particles via cryo-EM. Their study on the $30 \mathrm{~S}$ ribosomal subunit complexed with tRNA, mRNA and Initiation Factors (IFs) 1-3 elucidates how IF1 is positioned crucially to activate IF2 and IF3 consequently and similarly, how the position and orientation of an extended domain by IF2 is accurate enough to capture the formylmethionine charged on tRNA. This study clearly exhibits how major conformation changes of both IF3 and tRNA are noticeable for accommodating fMet-tRNAfMet in P-site and thereby allowing start codon recognition.

Next in line is the process of termination. Termination in protein synthesis occurs when a stop codon moves into the ribosomal decoding centre (DC) and is recognized by a class- 1 release factor (RF). RFs carry a conserved amino-acid motif, GGQ, which supposedly interacts directly with the peptidyltransferase centre (PTC) of the 50S ribosomal subunit and thus, is crucial for peptide release. There has also been a discussion among biologists about another conserved motif of RFs (SPF in RF2) which is proposed to interact directly with stop codons in the DC of the $30 \mathrm{~S}$ subunit. It was observed in the X-ray structure of RF2 that SPF and GGQ are only $23 \AA$ apart. Now, it should be remembered that the distance between the DC and PTC is $73 \AA$, which indicates that these two cannot be at DC and PTC simultaneously. This confusion was solved by Rawat et al. (2003) (48) who used a highly efficient in vitro system for bacterial protein synthesis and they created a release complex (RC) with ribosomes having a UAA stop codon in the A site and fMet-Phe-Thr- Ile-tRNAIle in the P site. Thus, it was evident that RF2 maintains an open conformation when bound to the ribosome and allows GGQ to reach the PTC while still allowing SPF-stop-codon interaction. Another release factor involved in this termination process is RF3, whose recruition is of essence to remove both RF1 and RF2 to carry out termination via GTP hydrolysis. Klaholz et al., 2004 (34) demonstrated the release mechanism of RFs with the help of cryo-EM which enabled them to visualize the RF3, bound to a nonhydrolysable analogue of GTP, in two statespre-bound to ribosome in one and accessing the GTPase centre in the next. With the translocation of tRNA to the exit site, major ribosomal rearrangements are bound to take place, which through this technique addresses the issue to understand the mechanism involved in carrying out translational termination. The results aided by cryo-EM were thus path breaking as new accuracy in the understanding of termination was achieved.

Deviating the focus to ribosomes obtained from other domains of life, significant contributions were done by Gomez et al. in 2000 (25) who highlighted the interaction of domain IV of EF2 with P-site bound tRNA via 3D map and molecular alignment and arrangement of eukaryotic ribosome-EF2 complex of 
Saccharomyces cerevisiae. The group even studied correlation of excess density of EF2domain IV to have interaction with 40 S subunit head and $60 \mathrm{~S}$ subunit bridge. Mammalian mitoribosome attracted attention of structural biologists to decipher their intricate machineries and specific activities. Kaushal et al., 2014 (22) utilized cryo-EM to solve the subunit structures of the mammalian ribosome only to discover 35 specific mitoribosomal proteins in special contact with inter-MRPs, indicative of their constructive organisation and characteristic evidences of evolution. The map also explains the involvement of the specific mitoribosomal proteins with both the subunits at the inter-subunit bridge.

Other than revealing structural facets of prokaryotic and eukaryotic ribosomes, cryo-EM even targetted to answer important questions as - what may be the cause for the limited use of antibiotics such as tetracycline and its everincreasing antibiotic resistance? Arenz et al. in 2015 (4) answered how this may be occurring by elucidating the structure of a ribosome protection protein, TetM at $3.9 \AA$ bound to a translating ribosome. This structure provides insights into the molecular physiology as to how TetM functions to dislodge the drug from its binding site on the ribosome.

Therefore, single particle cryo-EM though progressively made significant developments in structural biology providing high-resolution structure factors of macromolecular complexes and their intricate mechanistic details, as mentioned above, it was still highly non-uniform in local resolution, with most structures limited to above $3 \AA$ A resolutions, which was circumvented later on, leading to resolution revolution.

The cryo-EM structure of the $70 \mathrm{~S}$ ribosome, one of the ideal examples, from E. coli in complex with elongation factor $\mathrm{Tu}$, aminoacyl-tRNA and the antibiotic kirromycin has been already studied in greater detail and quite elaborately. Stark et al., 1997 (58) used cryo-EM to study the binding of EF-Tu to L7/L12 stalk of $50 \mathrm{~S}$ ribosomal subunit and thereby, the formation of a stable, 'ternary' complex along with amino-acyl tRNA and GTP to point out tRNA position on ribosome and its structure in pre and post-translocational states. But, the same complex with better resolutions at 2.65-2.9 ̊ using spherical aberration (Cs)corrected cryo-EM, as reported by Fischer et al. (2015) (16) along with the structures of the E. coli $70 \mathrm{~S}$ ribosome at $11.5 \AA$ and $50 \mathrm{~S}$ subunit at $7.5 \AA$ resolution $(22,41)$, respectively, now disclosed newer information which facilitated structure-function correlation and analyses. Fischer et al. (2015) (16) used aberrationcorrected cryo-EM in combination with extensive computational sorting to solve the structure of the E. coli $70 \mathrm{~S}$ ribosome at high resolution to visualize rRNA modifications and dynamic parts of the ribosome. Cryo-EM images were recorded in a $300 \mathrm{kV}$ electron cryomicroscope equipped with a sphericalaberration corrector and a direct electron detector operated in integration mode without alignment of intermediate image frames for motion correction. The aberration corrector was specifically tuned to reduce resolution-limiting aberrations and distortions. Overall, the cryoEM reconstruction at $2.9 \AA$ resolution is almost at par with the best-resolved X-ray structure of the E. coli $70 \mathrm{~S}$ ribosome $(2.8 \AA)$, but highlights and delineates further detailed information $(2.65 \AA)$ of the ribosomal core which holds significant relevance functionally and helps in elaborate mechanistic studies. The cryo-EM map turned out to be very effective not only to explain the structure of all 35 rRNA modifications in the bacterial ribosome for the first time but also clearly demonstrated how they moderate and fine-tune ribosome structure and function also giving a cue to the information of the action of antibiotics.

It is single particle cryo-EM that made it possible to even venture into sub-nanometer resolution structures such as that of the bacterial ribosome-SecYEG complex in a Nanodisc allowing for the determination of molecular make and model of the membrane protein, the SecYEG complex, in its natural habitat- the lipid bilayer environment. Molecular dynamics simulation studies along with visualization with cryo-EM reveal how the membrane may be party to the insertase activity of the protein-conducting channel by establishing steady and secured interactions between rRNA and the membrane. This method using high-resolution single particle cryo-EM likely can be taken up as a general approach in order to visualize functional membrane proteins in their respective lipid environments (20) and even for structural identification and analyses of particles other than ribosome.

Single particle cryo-EM, however, in the span of last 5 years, witnessed immense development in all technical aspects. The improvements discernible in instrumentation and computational image processing analysis, now revolutionized the scale of visualization to a greater extent unravelling finer details of complex macromolecular structures of ribosome (see Figure 4). 


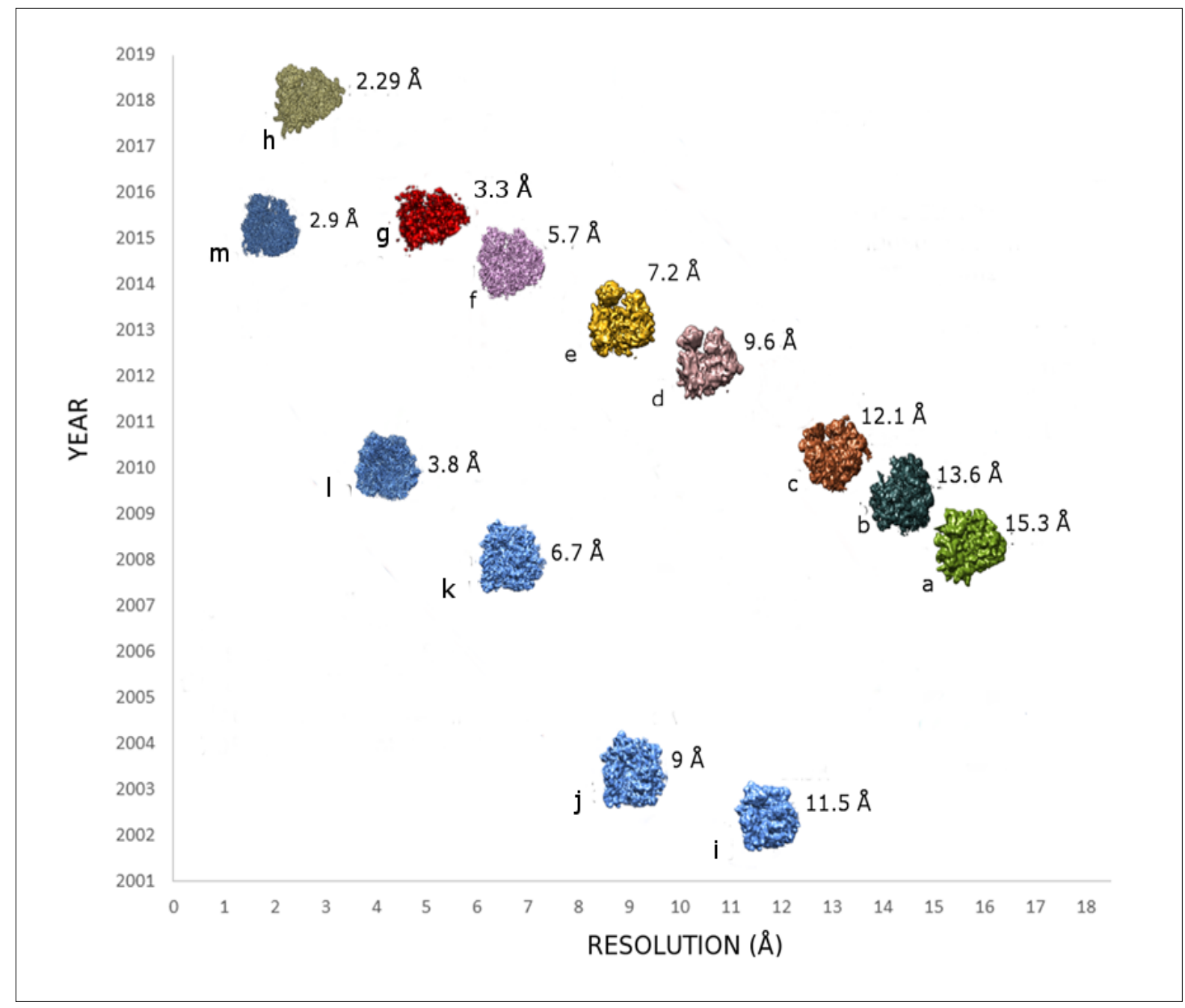

Figure 4: Advances in the resolution of single particle eukaryotic and prokaryotic ribosome structures through cryo-EM, in past few years.

(a-h) depicts $80 \mathrm{~S}$ ribosomes from various domains whereas, (i-m) represents $70 \mathrm{~S}$ E. coli ribosomes which are depicted in blue colour.

a: Thermomyces lanuginosus eEF2-80S Ribosome Transition State Complex; 15.3 Å; EMD5016; SPIDER; HAADF detector. (53)

b: Rabbit reticulocyte ribosome stalled on a stem-loop-containing mRNA with a P- site tRNA; 13.6 Ä; EMD-1670; SPIDER, WELLMAP; KODAK S0-163 FILM. (17)

c: Yeast $80 \mathrm{~S}$ ibosome stalled by a stem-loop containing mRNA; $12.1 \AA$; EMD- 1808 ;

SPIDER, WELLMAP; KODAK S0-163 FILM. (7)

$\mathrm{d}$ : Chaetomium thermophilum RAC bound to the $80 \mathrm{~S}$ ribosome; $9.6 \AA$ A; EMD-2179; SPIDER; TVIPS TEMCAM-F4 16 (4K*4k). (37)

e: 80S-RAC complex from yeast; 7.2 Å; EMD-6105; Relion; FHEAGLE $\quad$ (4K*4K).

f: Plasmodium falciparum 80S ribosome bound to P-tRNA; 6.7 Å; EMD-6456; Arachnid, CTFFIND3, Relion; GATAN K2(4K*4K). (58)

g: Mammalian ribosomal termination complex with accommodated eRF1; $3.75 \AA$; EMD-4132; Relion; FEI FALCON II (4K*4K). (56)

h: Human $80 \mathrm{~S}$ ribosome - (composite structure); $2.29 \AA$ A; EMD-3883; Relion2; FEI

FALCON II (4K*4K). (44) (Map released on 24.01.2018)

i: Solution structure of the E. coli $70 \mathrm{~S}$ ibosome; $11.5 \AA$ A; EMD-1003; SPIDER/WEB;

KODAK S0-163 FILM. (22) 
$\mathrm{j}$ : Incorporation of aminoacyl-tRNA into the ibosome in E. coli 70S ibosome; $9 \AA$ E.MD-1056; SPIDER; KODAK S0-163 FILM. (63)

k: Aminoacyl-tRNA-EF-Tu-GDP-kir ternary complex-bound E. coli $70 \mathrm{~S}$ ibosome; 6.7 Å; EMD-5036; SPIDER; KODAK S0-163 FILM. (65)

1: Nascent polypeptide chain-mediated translational stalling in E. coli $70 \mathrm{~S}$ ubosome; 5.8 Аं; EMD-1657; SPIDER; KODAK S0-163 FILM. (52)

m: E. coli ubosome-EF-Tu complex by Cs-corrected cryo-EM; 2.9 Å; EMD- 2847;

IMAGIC-5, Relion 1.2; FEI FALCON I (4K*4k). (16)

\section{The leap in the structural characterization: Ribosomes- the perfect example}

The strategy applied by Fischer et al. (2015) (16) to apply hierarchical classification proved to be effective in which the ribosome images were computationally sorted for the known modes of ribosomal motion. The potential sources of heterogeneity while preparing ribosomes, were also considered. Thus, the final cryo-EM map, which was of $2.9 \AA$ overall resolution, was subsequently used to refine the atomic model for the entire ribosome which included metal ions (Mg21, Zn21) and rRNA modifications. It was observed that the local resolution map, on the other hand, is showing relatively uniform resolution. The map showed resolution better than $2.9 \AA$ for most portions of the map, whereas only few parts of the ribosome, that too those portions which are located at the very periphery, are limited to resolutions- $3.5 \AA$. When considered in a local basis, it was determined by the crystallographic measures of FSC work (Fourier shell correlation) and CC work (Pearson correlation coefficient) that average resolutions were $2.8 \AA$ for $65 \%, 2.7 \AA$ for $44 \%$ and $2.65 \AA$ for $24 \%$ of the map . The final 3D map obtained was very much comparable with the available X-ray structures of the E. coli ribosome with a similar, or locally even better resolution obtained by cryo-EM. The structural attributes of side chains were also highly similar to the X-ray data available, as judged by local real-space correlations. In the best-defined areas of the map Mg21 ions, along with water molecules were visible in the coordination sphere, which indicates the achieved optical resolution was at least $2.8 \AA$.

It was also for the first time when modifications in rRNA was reported, absent even in high-resolution X-ray structures of the bacterial ribosome at 2.4-2.8 ̊ resolution, and were only observed in X-ray crystallographic images of the 50S subunit of Haloarcula marismortui at 2.2-2.4 A resolution. However, cryo-EM maps presented an incredible view of even single methyl groups of nucleosides along with non-planar base of dihydrouridine at position 2449 of 23S rRNA. Within hydrogen bonding distance of the N1 position, the cryoEM density map suggests presence of pseudouridines, indirectly indicated by polar residues. Overall, 35 constitutive rRNA modifications of the E. coli $70 \mathrm{~S}$ ribosome were thus mediated by high cryo-EM structures. (Fischer et al., 2015) (16)

Other than these, many examples are to follow which caused the leap. The improvements in techniques, microscopic apparatus and processing efficiencies of cryoEM, post 2013, led to a huge resolution revolution in structural biology. Ribosomes from all domains were being resolved at a resolution $<5 \AA$, unravelling important functional aspects of the molecule in various sectors, not known before. Of the numerous maps deposited below $5 \AA$, some of the vital ribosome molecules noted in different sectors are worth mentioning. Basilet et al. in 2014 (26) studied the markedly different mammalian $55 \mathrm{~S}$ mitochondrial ribosome with the help of cryo$\mathrm{EM}$, to reveal the detailed location and folding pattern of around 50 ribosomal subunits at 3.4 $\AA$. (see Figure 5). This highlights its conserved peptidyltransferase active site complexed with tRNAs for substrate transfer and defining the path for nascent peptide chain to follow. Improved image processing softwares such as RELION and EMAN played important roles in structure reconstruction and their better algorithmic functions took reconstruction and refinement of particles to a higher level. Using these softwares, Khatter et al. in 2015 (32) elucidated the structure of 


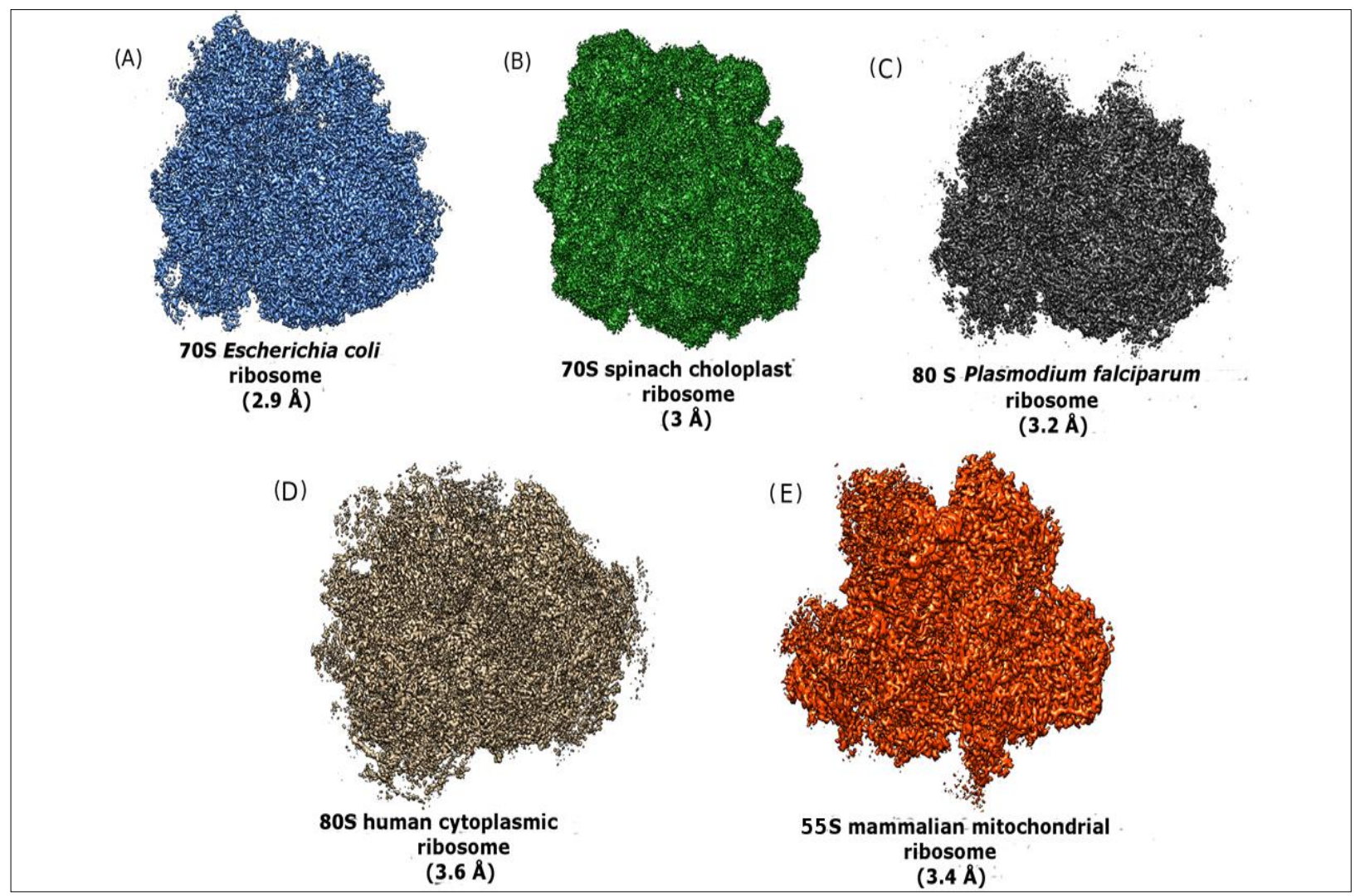

Figure 5: A panel depicting high resolution single particle reconstructed images of ribosome from various domains through cryo-EM.

(A) Prokaryotic 70S ribosome of E. coli complexed with EF-Tu; $2.9 \AA$; EMD-2847.

(B) 70S Spinach chloroplast ribosome; $3 \AA$; EMD-3941. (9)

(C) 80S Ribosome of Plasmodium falciparum pathogen; $3.2 \AA$; EMD-2660.

(D) Eukaryotic 80S human cytoplasmic ribosome; $3.6 \AA ̊$; EMD-2938. (32)

(E) $55 \mathrm{~S}$ ribosome of mammalian mitochondria; $3.4 \AA$; EMD-2787. (26)

eukaryotic 80S human ribosome at a high resolution of $2.9 \AA$. The structure divulges unprecedented information regarding rRNA moieties, amino acid side chains, the tRNA binding locales and essentially the molecular interaction involved in tRNA exit and even Natchiar et al., 2017 (44) successfully showcased the intricate machineries in their eukaryotic 80S human ribosome composite structure at $2.29 \AA$, which is by far the best resolution obtained in case of ribosomes in all domains of life. Detailed modelling of the subunit interface paves the way to further analyse antibiotic side effects and the cause of the diseases induced by deregulated protein synthesis (see Figure 5).
Other ribosomal structures also came to the foreground, during this time, highlighting the essentiality of knowing the intricate mechanisms regulating them which further helps to analyse how structural changes influence and control functional aspects of any particle, under consideration. Spinach chloroplast ribosome with chl-RRF and hibernation promoting factor (Figure 5), studied by Boerema et al., 2018 (9) at $3 \AA$ A provides new insights to chloro-ribosome's structural and evolutionary aspects which remained elusive prior to this. This refined structure reveals structural rearrangements and existence of a tunnel extending till the ribosome exterior. 
Another landmark in the cryo-EM field came about with the onset of Time Resolved Cryo-EM, which has the potential to capture, reconstruct and analyse intermittent steps, otherwise lost in standard lengthy cryo-EM technique or other structural biology techniques (19). The process addresses those molecules which are not in equilibrium and need to pass through several intermediate conformations to reach equilibrium ultimately. As seen by Zavialov et al., 2005 (69), termination of translation is followed by splitting of the ribosomes into its subunits with subsequent binding of EF-G.GTP and Ribosome Recycling Factor, hydrolysing GTP on EF-G of post-termination complex of ribosome. Fu et al., 2016 (21) described that when they started to collect image pictures of the mixed sample of PostTC.RRF with EF-G.GTP, four distinct intermediate, short-lived subpoulation namely PostTC.RRF, PostTC.RRF.EF-G.GTP, 50S subunit, and $30 \mathrm{~S}$ subunit were observed via Time Resolved Cryo-EM as expected along with other variant subpopulations of ligand bound $30 \mathrm{~S}$ and 50S subunits, when sorted and reconstructed using RELION, thus demonstrating the power of this technique to even capture the extreme short-lived intermediates. The most interesting and important thus far, among the intermediates as resolved using this technique appears to be PostTC.RRF-EF-G complex which depicts how EF-G binding and hydrolysis of GTP is one of the causes for intersubunit rotation and how it forces the domain II of Recycling factor to pivot towards the intersubunit bridge- the one responsible to hold the association of 30S-50S stably. This depicts thereby how the separation of subunits for consequent recycling process actually takes place. This process also opens the gateway to understand the fate of tRNA and the role of various subunits in rotational and conformational changes to carry through the task of translation. This process also clears the air for any ambiguity regarding the role of IF3 in recycling, thus putting an end to many controversies over the past years.

Therefore, it can be clearly concluded that cryo-EM is highly efficient to visualise and analyse significant dynamic macromolecular machineries such as the ribosome. In most of the recent works, the obtained resolution at a uniform level is achieved better than $3 \AA$ which is really promising for detailed analyses. Reconstruction from electron microscopic images by computation has turned out to be as powerful as X-ray crystallisation technique to elucidate ribosome conformation and composition. It has now been possible to visualise ribosome structures with bound water molecules, ions and rRNA modifications as well as the side chain residues. Thus cryo-EM provides structural biologists deep insights into ribosome structure and its ligand interaction which further becomes a tool to develop new antibiotics against pathogenic agents.

Technological advancements have therefore seen a tremendous leap in the ribosome structures from every domain in the last 10 years, providing illustrious insights into their intricate core mechanisms with high nearatomic resolutions. This mediates the cryo-EM to be a useful tool even for therapeutics, for identifying and investigating broad spectrum of drug-target interactions, drug delivery studies and dynamicity of particles' conformational states. Hentschel et al., 2017 (72) studied the $70 \mathrm{~S}$ ribosome of Mycobacterium smegmatis, one of the known agents of tuberculosis. Their cryoEM study of the ribosome complex reveals two additional ribosomal proteins in vicinity of the probable drug target sites, both in catalytic and decoding centre of ribosome. Specifically, rRNA and protein expansions are noticed which remodelled the surface of ribosome, implicating polysome organisation. This structure thus exploits the basic understanding of Mycobacterium translational mechanism in minute detail correlating with atomic structure and facilitating therapeutics for tuberculosis. The structure of emetine drug bound ribosome of Plasmodium falciparum at $3.2 \AA$ reveals the mechanism of interaction of this anti-protozoan drug (67), (see Figure 6) which actually paved the way for further detailed studies regarding the efficacy of the drug and its targeting system. 


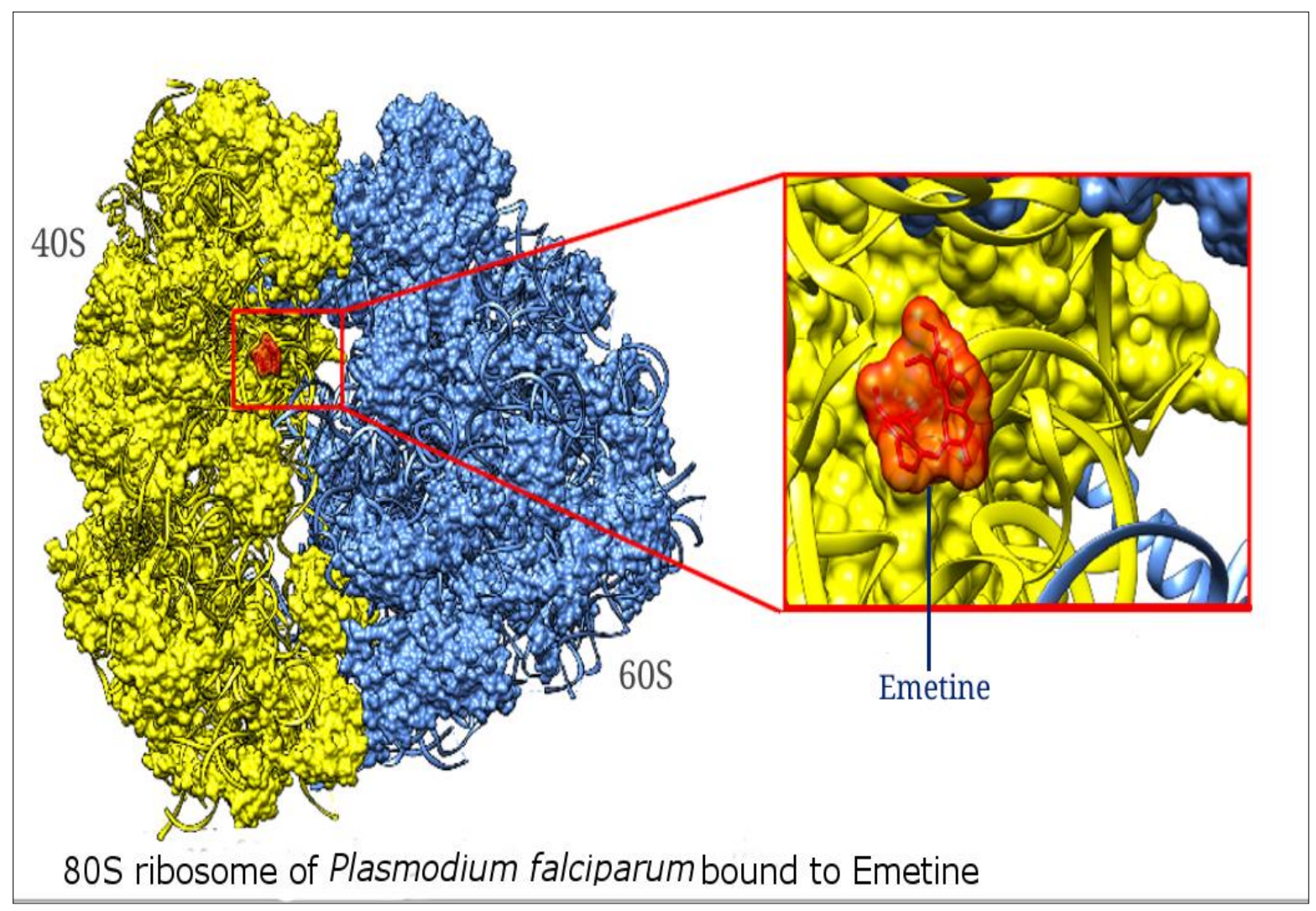

Figure 6: 80S ribosome of Plasmodium falciparum visualized at $3.2 \AA$ bound to the antiprotozoan drug Emetine. The yellow coloured surface is $40 \mathrm{~S}$ protein density subunit, whereas the blue colour represents $60 \mathrm{~S}$ protein density subunit.

Inset depicts the Emetine drug bound to the ribosomal subunit of the Plasmodium falciparum ribosomal complex.

Shalev-Benami et al., 2017 (55) even successfully depicted the site for the binding of the drug paromomycin to Leishmania donovanii large subunit of ribosomal complex. Merk et al. (2016) have shown how $3.8 \AA$ high resolution cryo-EM structural elucidation of the cancer target isocitrate dehydrogenase, a small, dynamic protein complex have helped in studying its dynamic mechanism and conformational changes that can be induced by another small-molecule, an allosteric inhibitor ML309. They have also recorded $2.8 \AA$ and $1.8 \AA$ high resolutions, near-atomic level reconstructions of lactate dehydrogenase and glutamate dehydrogenase complexes respectively, paving way for further drug targeted research. Focussing on ribosome, cryoEM has advanced to such extents that it can even dive inside the mechanistic properties of the ribosomal complex and elucidate structures which explained translational inhibition and stalling. As reported in 2014 by Arenz and group (3), Staphylococcus aureus ErmCL leader peptide on interaction with macrolide antibiotics like erythromycin, which may bind to the tunnel of the ribosome, induces certain allosteric conformational changes, thereby making rearrangement at the peptidyltransferase centre (PTC) of the ribosome. This definitely causes disturbances at the PTC and creates instability in the binding of tRNA to the amino-acyl site of the ribosomestalling the translational process and affecting the regulation of expression of certain downstream genes. This entire process has been analysed simply from the depiction of the ErmCL-stalled ribosomal unit reconstitution by cryo-EM imaging at 3.9 Å. Thus, cryo-EM created a stir in the medical and research fields by helping in analysing the efficacy with which the bound ligands can interact providing a glimpse of drug delivery and its mode of action. 


\section{Current Standpoint}

With the advancement in technical aspects, cryo-EM currently has the capability to solve de novo structures of ribosomes from any domain, with no reference map fit required to X-ray crystal structures or NMR structures. And with these, resolutions below and approaching to $5 \AA$ can be achieved as discussed above. Moreover, with the refined and improved graphics for modelling and animations, softwares like COOT (15), CHIMERA (46) and REFMAC (66) were used widely as in cases of X-ray crystal structures making visualization a treat.

Less noise leads to less motion blurring and higher order homogenous data set leading to determination of each particle accurately and that can be achieved now by cryo-EM with the advent of the Direct electron detectors, making work with heterogenous samples less cumbersome. Thus, images with stronger signals directly correlating to lower noise and higher resolution maps, in synergy, albeit with the help of image processors like EMAN, RELION, FREALIGN, SPIDER, IMAGIC and others, various categories of ribosomes were reconstituted at even below 5 Å resolution.

In order for visualisation towards smaller molecules, though a step behind from de novo reconstruction, via assisted fold-symmetry and/or homologous structural reference, cryoEM can still position polyamino acids correctly with progressive on-point assignments.

\section{The path in future}

Sectors for more developments and improvements lie in production of thinner

\section{References}

1. Agrawal RK, Heagle AB, Frank J. Studies of elongation factor G-dependent tRNA translocation by three-dimensional cryo-electron microscopy. InThe Ribosome 2000 Jan 1 (pp. 53-62). American Society of Microbiology.

2. Amunts A, Brown A, Bai XC, Llácer JL, Hussain T, Emsley P, Long F, Murshudov G, Scheres $\mathrm{SH}$, Ramakrishnan V. Structure of the yeast mitochondrial large ribosomal subunit. Science. 2014 Mar 28;343(6178):1485-9.

3. Arenz $\mathrm{S}$, Meydan $\mathrm{S}$, Starosta $\mathrm{AL}$, Berninghausen 0, Beckmann R, Vázquez-Laslop N, Wilson DN. Drug sensing by the ribosome induces translational arrest via active site perturbation. Molecular cell. 2014 Nov 6;56(3):446-52.

4. Arenz S, Nguyen F, Beckmann R, Wilson DN. Cryo-EM structure of the tetracycline resistance detectors, better motion correction algorithms having better counting and faster read-outs. Beam induced motions though significantly corrected, there can still be room for efficient corrections; where the sample if not allowed to move at all can lead to a huge accomplishmenta novel data collection method yielding better image categorization, reduce noise:signal ratio, better coping mechanism with heterogeneity and all these cumulatively will actually save a lot of time and moreover may unravel various facets of the ribosomal world yet unknown. Progress though achieved with high-throughput images and animations, the time and expenses involved is immense. Parallel improvements with mutual efforts in academia and industry can actually let cryo-EM thrive in and revolutionize the field of structural biology like never before, as the advancement is clearly visible via Figure 1. Ribosome is an essential part of the transcriptomics. When it boils down to analyse structure to function correlations, ribosome has played crucial roles almost always. To back that, higher resolution images can actually be an insightful step to a breakthrough. Additionally, ribosomes represent asymmetric population of samples. It reflects the technological advancements in cryoEM to solve the innate structural mysteries of asymmetric particles.

\section{Author contribution}

The idea was conceptualized by AA. The manuscript was written by AA \& AC.

\section{Conflict of Interest}

The authors declare to have no conflicts of interest for the contents of this article.

protein TetM in complex with a translating ribosome at $3.9-\AA$ resolution. Proceedings of the National Academy of Sciences. 2015 Apr 28;112(17):5401-6.

5. Bai XC, McMullan G, Scheres SH. How cryoEM is revolutionizing structural biology. Trends in biochemical sciences. 2015 Jan 1;40(1):49-57.

6. Bartesaghi A, Merk A, Banerjee S, Matthies D, Wu X, Milne JL, Subramaniam S. 2.2 Å resolution cryoEM structure of $\beta$-galactosidase in complex with a cell-permeant inhibitor. Science. 2015 Jun 5;348(6239):1147-51.

7. Becker T, Armache JP, Jarasch A, Anger AM, Villa E, Sieber H, Motaal BA, Mielke T, Berninghausen O, Beckmann R. Structure of the no-go mRNA decay complex Dom34-Hbs1 bound to a stalled 80S ribosome. Nature structural \& molecular biology. 2011 Jun;18(6):715. 
8. Belnap DM. Electron microscopy and image processing: essential tools for structural analysis of macromolecules. Current protocols in protein science. 2015 Nov;82(1):17-2.

9. Boerema AP, Aibara S, Paul B, Tobiasson V, Kimanius D, Forsberg BO, Wallden K, Lindahl E, Amunts A. Structure of the chloroplast ribosome with chl-RRF and hibernation-promoting factor. Nature plants. 2018 Apr;4(4):212-7.

10. Carter AP, Clemons WM, Brodersen DE, Morgan-Warren RJ, Wimberly BT, Ramakrishnan V. Functional insights from the structure of the $30 \mathrm{~S}$ ribosomal subunit and its interactions with antibiotics. Nature. 2000 Sep;407(6802):340-8.

11. Cheng Y. Single-particle cryo-EM at crystallographic resolution. Cell. 2015 Apr 23;161(3):450-7.

12. Datta PP, Sharma MR, Qi L, Frank J, Agrawal RK. Interaction of the $G^{\prime}$ domain of elongation factor $G$ and the C-terminal domain of ribosomal protein L7/L12 during translocation as revealed by cryo-EM. Molecular cell. 2005 Dec 9;20(5):723-31.

13. De la Rosa-Trevín JM, Otón J, Marabini R, Zaldivar A, Vargas J, Carazo JM, Sorzano CO. Xmipp 3.0: an improved software suite for image processing in electron microscopy. Journal of structural biology. 2013 Nov 1;184(2):321-8.

14. Dubochet J, Booy FP, Freeman R, Jones AV, Walter CA. Low temperature electron microscopy. Annual review of biophysics and bioengineering. 1981 Jun;10(1):133-49.

15. Emsley P, Cowtan K. Coot: model-building tools for molecular graphics. Acta Crystallographica Section D: Biological Crystallography. 2004 Dec 1;60(12):2126-32.

16. Fischer N, Neumann P, Konevega AL, Bock LV, Ficner R, Rodnina MV, Stark H. Structure of the E. coli ribosome-EF-Tu complex at $<3 \AA$ resolution by $\mathrm{C}$ s-corrected cryo-EM. Nature. 2015 Apr;520(7548):567-70.

17. Flanagan IV JF, Namy O, Brierley I, Gilbert RJ. Direct observation of distinct A/P hybrid-state tRNAs in translocating ribosomes. Structure. 2010 Feb 10;18(2):257-64.

18. Frank J. Cryo-electron microscopy as an investigative tool: the ribosome as an example. Bioessays. 2001 Aug;23(8):725-32.

19. Frank J. Time-resolved cryo-electron microscopy: Recent progress. Journal of structural biology. 2017 Dec 1;200(3):303-6.

20. Frauenfeld J, Gumbart J, Van Der Sluis EO, Funes S, Gartmann M, Beatrix B, Mielke T, Berninghausen O, Becker T, Schulten K, Beckmann R. Cryo-EM structure of the ribosome-SecYE complex in the membrane environment. Nature structural \& molecular biology. 2011 May;18(5):614.

21. Fu Z, Kaledhonkar S, Borg A, Sun M, Chen B, Grassucci RA, Ehrenberg M, Frank J. Key intermediates in ribosome recycling visualized by time-resolved cryoelectron microscopy. Structure. 2016 Dec 6;24(12):2092-101.

22. Gabashvili IS, Agrawal RK, Spahn CM,
Grassucci RA, Svergun DI, Frank J, Penczek P. Solution structure of the E. coli $70 \mathrm{~S}$ ribosome at $11.5 \AA$ resolution. Cell. 2000 Mar 3;100(5):537-49.

23. Ge P, Zhou ZH. Hydrogen-bonding networks and RNA bases revealed by cryo electron microscopy suggest a triggering mechanism for calcium switches. Proceedings of the National Academy of Sciences. 2011 Jun 7;108(23):9637-42.

24. Gilbert RJ, Fucini P, Connell S, Fuller SD, Nierhaus KH, Robinson CV, Dobson CM, Stuart DI. Three-dimensional structures of translating ribosomes by Cryo-EM. Molecular cell. 2004 Apr 9;14(1):57-66.

25. Gomez-Lorenzo MG, Spahn CM, Agrawal RK, Grassucci RA, Penczek P, Chakraburtty K, Ballesta JP, Lavandera JL, Garcia-Bustos JF, Frank J. Three-dimensional cryo-electron microscopy localization of EF2 in the Saccharomyces cerevisiae $80 \mathrm{~S}$ ribosome at $17.5 \AA$ resolution. The EMBO journal. 2000 Jun 1;19(11):2710-8.

26. Greber BJ, Bieri P, Leibundgut M, Leitner A, Aebersold R, Boehringer D, Ban N. The complete structure of the $55 \mathrm{~S}$ mammalian mitochondrial ribosome. Science. 2015 Apr 17;348(6232):303-8.

27. Halic M, Becker T, Pool MR, Spahn CM, Grassucci RA, Frank J, Beckmann R. Structure of the signal recognition particle interacting with the elongation-arrested ribosome. Nature. 2004 Feb;427(6977):808-14.

28. Heymann JB. Bsoft: image and molecular processing in electron microscopy. Journal of structural biology. 2001 Feb 1;133(2-3):156-69.

29. Hussain T, Llácer JL, Wimberly BT, Kieft JS, Ramakrishnan V. Large-scale movements of IF3 and tRNA during bacterial translation initiation. Cell. 2016 Sep 22;167(1):133-44.

30. Jomaa A, Boehringer D, Leibundgut M, Ban N. Structures of the E. coli translating ribosome with SRP and its receptor and with the translocon. Nature communications. 2016 Jan 25;7(1):1-9.

31. Kaushal PS, Sharma MR, Booth TM, Haque EM, Tung CS, Sanbonmatsu KY, Spremulli LL, Agrawal RK. Cryo-EM structure of the small subunit of the mammalian mitochondrial ribosome. Proceedings of the National Academy of Sciences. 2014 May 20;111(20):7284-9.

32. Khatter H, Myasnikov AG, Natchiar SK, Klaholz BP. Structure of the human 80S ribosome. Nature. 2015 Apr;520(7549):640-5.

33. Kim J, Wu S, Tomasiak TM, Mergel C, Winter MB, Stiller SB, Robles-Colmanares Y, Stroud RM, Tampé R, Craik CS, Cheng Y. Subnanometreresolution electron cryomicroscopy structure of a heterodimeric ABC exporter. Nature. 2015 Jan;517(7534):396-400.

34. Klaholz BP, Myasnikov AG, van Heel M. Visualization of release factor 3 on the ribosome during termination of protein synthesis. Nature. 2004 Feb;427(6977):862-5.

35. Kühlbrandt W. Microscopy: cryo-EM enters a new era. Elife. 2014 Aug 13;3:e03678.

36. Kühlbrandt W. The resolution revolution. 
Science. 2014 Mar 28;343(6178):1443-4.

37. Leidig C, Bange G, Kopp J, Amlacher S, Aravind A, Wickles S, Witte G, Hurt E, Beckmann R, Sinning I. Structural characterization of a eukaryotic chaperone-the ribosome-associated complex. Nature structural \& molecular biology. 2013 Jan;20(1):23.

38. Li X, Mooney P, Zheng S, Booth CR, Braunfeld MB, Gubbens S, Agard DA, Cheng Y. Electron counting and beam-induced motion correction enable nearatomic-resolution single-particle cryo-EM. Nature methods. 2013 Jun;10(6):584.

39. Ludtke SJ, Baldwin PR, Chiu W. EMAN: semiautomated software for high-resolution singleparticle reconstructions. Journal of structural biology. 1999 Dec 1;128(1):82-97.

40. Malhotra A, Penczek P, Agrawal RK, Gabashvili IS, Grassucci RA, JuÈnemann R, Burkhardt $\mathrm{N}$, Nierhaus KH, Frank J. Escherichia coli $70 \mathrm{~S}$ ribosome at $15 \AA$ resolution by cryo-electron microscopy: localization of fMet-tRNAfMet and fitting of L1 protein. Journal of molecular biology. $1998 \mathrm{Jul}$ 3;280(1):103-16.

41. Matadeen R, Patwardhan A, Gowen B, Orlova EV, Pape T, Cuff M, Mueller F, Brimacombe R, van Heel M. The Escherichia coli large ribosomal subunit at 7.5 Å resolution. Structure. 1999 Jan 1;7(12):1575-83.

42. Merk A, Bartesaghi A, Banerjee S, Falconieri V, Rao P, Davis MI, Pragani R, Boxer MB, Earl LA, Milne JL, Subramaniam S. Breaking cryo-EM resolution barriers to facilitate drug discovery. Cell. 2016 Jun 16;165(7):1698-707.

43. Mitra K, Frank J. Ribosome dynamics: insights from atomic structure modeling into cryoelectron microscopy maps. Annu. Rev. Biophys. Biomol. Struct.. 2006 Jun 9;35:299-317.

44. Natchiar SK, Myasnikov AG, Kratzat $H$, Hazemann I, Klaholz BP. Visualization of chemical modifications in the human $80 \mathrm{~S}$ ribosome structure. Nature. 2017 Nov;551(7681):472-7.

45. Nogales E. The development of cryo-EM into a mainstream structural biology technique. Nature methods. 2016 Jan;13(1):24-7.

46. Pettersen EF, Goddard TD, Huang CC, Couch GS, Greenblatt DM, Meng EC, Ferrin TE. UCSF Chimera-a visualization system for exploratory research and analysis. Journal of computational chemistry. 2004 Oct;25(13):1605-12.

47. Ramakrishnan V. Ribosome structure and the mechanism of translation. Cell. 2002 Feb 22;108(4):557-72.

48. Rawat UB, Zavialov AV, Sengupta J, Valle M, Grassucci RA, Linde J, Vestergaard B, Ehrenberg M, Frank J. A cryo-electron microscopic study of ribosome-bound termination factor RF2. Nature. 2003 Jan;421(6918):87-90.

49. Ruska H. Über ein neues bei der bakteriophagen Lyse auftretendes Formelement. Naturwissenschaften. 1941 Jun 1;29(24-25):367-8.

50. Scheres SH. RELION: implementation of a Bayesian approach to cryo-EM structure determination. Journal of structural biology. 2012 Dec
1;180(3):519-30.

51. Wong W, Bai XC, Brown A, Fernandez IS, Hanssen E, Condron M, Tan YH, Baum J, Scheres SH. Cryo-EM structure of the Plasmodium falciparum $80 \mathrm{~S}$ ribosome bound to the anti-protozoan drug emetine. Elife. 2014 Jun 9;3:e03080.

52. Seidelt B, Innis CA, Wilson DN, Gartmann M, Armache JP, Villa E, Trabuco LG, Becker T, Mielke T, Schulten K, Steitz TA. Structural insight into nascent polypeptide chain-mediated translational stalling. Science. 2009 Dec 4;326(5958):1412-5.

53. Sengupta J, Nilsson J, Gursky R, Kjeldgaard M, Nissen P, Frank J. Visualization of the eEF2-80S ribosome transition-state complex by cryo-electron microscopy. Journal of molecular biology. 2008 Sep 26;382(1):179-87.

54. Settembre EC, Chen JZ, Dormitzer PR, Grigorieff N, Harrison SC. Atomic model of an infectious rotavirus particle. The EMBO journal. 2011 Jan 19;30(2):408-16.

55. Shalev-Benami M, Zhang Y, Rozenberg H, Nobe Y, Taoka M, Matzov D, Zimmerman E, Bashan A, Isobe $\mathrm{T}$, Jaffe $\mathrm{CL}$, Yonath $\mathrm{A}$. Atomic resolution snapshot of Leishmania ribosome inhibition by the aminoglycoside paromomycin. Nature communications. 2017 Nov 17;8(1):1-9.

56. Shao S, Murray J, Brown A, Taunton J, Ramakrishnan V, Hegde RS. Decoding mammalian ribosome-mRNA states by translational GTPase complexes. Cell. 2016 Nov 17;167(5):1229-40.

57. Skiniotis G, Southworth DR. Single-particle cryo-electron microscopy of macromolecular complexes. Microscopy. 2016 Feb 1;65(1):9-22.

58. Stark H, Rodnina MV, Rinke-Appel J, Brimacombe R, Wintermeyer $\mathrm{W}$, van Heel $M$. Visualization of elongation factor $\mathrm{Tu}$ on the Escherichia coli ribosome. Nature. 1997 Sep;389(6649):403-6.

59. Sun M, Li W, Blomqvist K, Das S, Hashem Y, Dvorin JD, Frank J. Dynamical features of the Plasmodium falciparum ribosome during translation. Nucleic acids research. 2015 Dec 2;43(21):10515-24.

60. TAMURA H. A transportable image processing software package: SPIDER. InProc. 6th Int. Joint Conf. on Pattern Recognition, 19821982.

61. Thompson RF, Walker M, Siebert CA, Muench SP, Ranson NA. An introduction to sample preparation and imaging by cryo-electron microscopy for structural biology. Methods. 2016 May 1;100:3-15. 62. Valle M, Sengupta J, Swami NK, Grassucci RA, Burkhardt N, Nierhaus KH, Agrawal RK, Frank J. Cryo-EM reveals an active role for aminoacyl-tRNA in the accommodation process. The EMBO journal. 2002 Jul 1;21(13):3557-67.

63. Valle M, Zavialov A, Li W, Stagg SM, Sengupta J, Nielsen RC, Nissen P, Harvey SC, Ehrenberg M, Frank J. Incorporation of aminoacyl-tRNA into the ribosome as seen by cryo-electron microscopy. Nature structural \& molecular biology. 2003 Nov;10(11):899-906.

64. van Heel M, Keegstra W. IMAGIC: a fast, flexible and friendly image analysis software system. 
Ultramicroscopy. 1981 Jan 1;7(2):113-29.

65. Villa E, Sengupta J, Trabuco LG, LeBarron J, Baxter WT, Shaikh TR, Grassucci RA, Nissen P, Ehrenberg M, Schulten K, Frank J. Ribosome-induced changes in elongation factor Tu conformation control GTP hydrolysis. Proceedings of the National Academy of Sciences. 2009 Jan 27;106(4):1063-8.

66. Winn MD, Murshudov GN, Papiz MZ. Macromolecular TLS refinement in REFMAC at moderate resolutions. InMethods in enzymology 2003 Jan 1 (Vol. 374, pp. 300-321). Academic Press.

67. Wong W, Bai XC, Brown A, Fernandez IS, Hanssen E, Condron M, Tan YH, Baum J, Scheres SH. Cryo-EM structure of the Plasmodium falciparum $80 \mathrm{~S}$ ribosome bound to the anti-protozoan drug emetine. Elife. 2014 Jun 9;3:e03080.

68. Yu X, Jin L, Zhou ZH. $3.88 \AA$ structure of cytoplasmic polyhedrosis virus by cryo-electron microscopy. Nature. 2008 May;453(7193):415-9.

69. Zavialov AV, Hauryliuk VV, Ehrenberg M. Splitting of the posttermination ribosome into subunits by the concerted action of RRF and EF-G.
Molecular cell. 2005 Jun 10;18(6):675-86.

70. Zhang X, Jin L, Fang Q, Hui WH, Zhou ZH. 3.3 $\AA$ cryo-EM structure of a nonenveloped virus reveals a priming mechanism for cell entry. Cell. $2010 \mathrm{Apr}$ 30;141(3):472-82.

71. Zhang Y, Ma C, Yuan Y, Zhu J, Li N, Chen C, $\mathrm{Wu} \mathrm{S}, \mathrm{Yu} \mathrm{L}$, Lei J, Gao N. Structural basis for interaction of a cotranslational chaperone with the eukaryotic ribosome. Nature structural \& molecular biology. 2014 Dec;21(12):1042.

72. Hentschel J, Burnside C, Mignot I, Leibundgut $\mathrm{M}$, Boehringer D, Ban N. The complete structure of the Mycobacterium smegmatis $70 \mathrm{~S}$ ribosome. Cell reports. 2017 Jul 5;20(1):149-60. 